Type of the Paper (Article)

\title{
Impact of using CSS PHY and RTS/CTS Combined with Frame Concatenation in the IEEE 802.15.4 Non- beacon Enabled Mode Performance
}

\author{
Norberto Barroca ${ }^{1}$, Luís M. Borges ${ }^{1}$, Fernando J. Velez ${ }^{1, *}$ and Periklis Chatzimisios ${ }^{2}$ \\ 1 Instituto de Telecomunicações and Universidade da Beira Interior, DEM - Faculdade de Engenharia, 6201- \\ 001 Covilhã, Portugal; fjv@ubi.pt \\ 2 International Hellenic University, School of Science \& Technology, Greece; pchatzimisios@ihu.gr \\ * Correspondence: fjvl@ubi.pt; Tel.: +351-275-329-953
}

\begin{abstract}
This paper studies the performance improvement for the nonbeacon-enabled mode of IEEE 802.15.4 originated by the inclusion of the Request-To-Send/Clear-To-Send (RTS/CTS) handshake mechanism combined with frame concatenation. Under IEEE 802.15.4 employing RTS/CTS, the backoff procedure is not repeated for each data frame sent but only for each RTS/CTS set. The throughput and delay performance are mathematically derived for both the Chirp Spread Spectrum and Direct Sequence Spread Spectrum Physical layers for the $2.4 \mathrm{GHz}$ band. Results show that the utilization of RTS/CTS significantly enhances the performance of IEEE 802.15.4 in terms of maximum throughput, minimum delay and bandwidth efficiency.
\end{abstract}

Keywords: Wireless Sensor Networks; MAC sub-layer; RTS/CTS/ non-beacon mode; packet concatenation; channel use; IEEE 802.15.4

\section{Introduction}

IEEE 802.15.4, which defines both Physical (PHY) and Medium Access Control (MAC) layers, is the de-facto communication standard [1], [2] providing low-power and low-data-rate communication for Wireless Personal Area Networks (WPANs). Various Working Groups within IEEE 802.15.4 are currently putting great efforts on developing new spectrum resource usage mechanisms or include the best of the best already existing ones for WPANs at Industrial, Scientific and Medical (ISM) and unlicensed bands [2]. The idea is to respond to the demands of the evolution of WSN applications, offering higher data rates when needed.

The Request-To-Send/Clear-To-Send (RTS/CTS) scheme has not been considered in any of the existing IEEE 802.15 standards but facilitates to shorten the duration of frame collisions, as shown in [3]. The proposed scheme involves the exchange of short RTS and CTS control frames prior to the exchange of the actual data frames. The fields of application from WSNs where the use of RTS/CTS assumes particular importance includes manufactory, healthcare and augmented reality, for which there is a need of sharing bursts of information with low collision probability. Although the proposal of employing RTS/CTS is not new and has already been standardized and implemented in legacy Wi-Fi (since it shortens packet collision duration, as shown in [3]), this reservation scheme has not been considered in any of the existing IEEE 802.15.4 standards. The research developed in the context of this work shows that inclusion of the RTS/CTS mechanism significantly improves network performance, clearly demonstrating that its omission is not beneficial for the IEEE 802.15.4 standards.

This paper evaluates the performance of the nonbeacon-enabled mode of the IEEE 802.15.4 MAC sub-layer improvement by employing the RTS/CTS handshake scheme combined with frame concatenation. Wireless nodes use equal backoff procedure from the IEEE 802.15.4 basic access mode. 
Nonetheless, this procedure is only repeated for each RTS/CTS set and not for each individual data frame. As such, channel utilization is optimized by decreasing the deferral time before transmitting each data frame. Differently from [4], in this paper, this performance enhancement is studied both for the Chirp Spread Spectrum (CSS) and Direct Sequence Spread Spectrum (DSSS) PHY that operate in the $2.4 \mathrm{GHz}$ Industrial, Scientific and Medical band.

The remaining of this paper is organized as follows. Section 2 starts by addressing the state transition diagram for the switching between radio states and describes the PHY protocol data unit. Then, the steps to calculate the bit error rate (BER), packet success rate (PSR) and packet error rate (PER) are presented, followed by the study of the variation of BER and PER with the Signal-to-Noise Ratio (SNR). The coexistence between IEEE 802.15.4 and other wireless system technologies that operate in the shared industrial, scientific and medical (ISM) band is discussed as well. Section 3 explores the mathematical formulations for time delays after addressing aspects of the MAC sublayer and control messages flowchart for the nonbeacon-enabled mode of IEEE 802.15.2. Section 4 presents the MAC sub-layer system model for the minimum delay and maximum throughput. Section 5 presents results for the system model metrics and bandwidth efficiency, and extracts lessons from the comparison between the CSS and DSSS PHY. Finally, conclusions are drawn in Section 6.

\section{Aspects of PHY layer}

The PHY layer is responsible for providing status information for the MAC sub-layer, switch between different radio states (i.e., RX, TX and SLEEP), send/receive/listen packets to/from channel, provide hooks for statistical information and configurable settings. The information can be passively (e.g., current channel state) or actively provided to the MAC layer (e.g., packet transmission complete) based on events. Some information must be provided on demand, as follows:

- Channel state (busy/idle) and Received Signal Strength Indicator (RSSI);

- Current radio state (e.g., RX, TX and SLEEP);

- Control information (e.g., transmission over and send).

Figure 1 presents the state transition diagram for switching between the different radio states. The time for switching mainly depends of the IEEE 802.15.4 compliant transceivers.

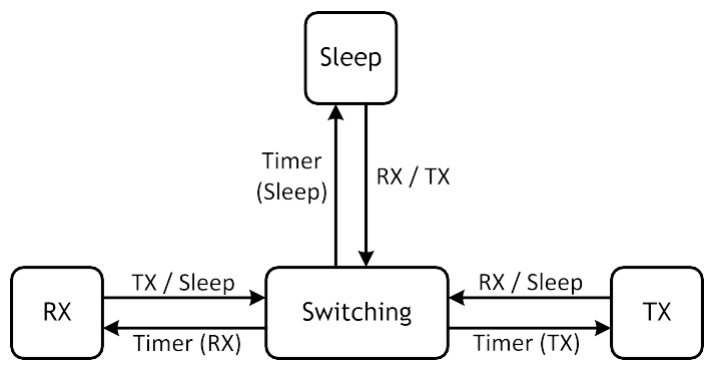

Figure 1. State transition diagram for the switching between different radio states.

In order to maintain a simple interface, both MAC and PHY layers share a common packet structure, as presented in Figure 2. This type of packet is known as the PHY Protocol Data Unit (PPDU). It is responsible for the encapsulation of all data structures from the higher layers of the protocol, and is divided into three basic components: Synchronization Header (SHR), PHY Header (PHR) and variable length payload, which contains the PHY Service Data Unit (PSDU) as follows:

- The SHR consists basically of two fields, a preamble sequence and a start of frame de- limiter. The preamble field has a length of 4 bytes, allowing for achieving chip and bit synchronization. The Start of Frame Delimiter (SFD) has a length of 1 byte and allows a receiver to set up the beginning of a packet; 
- The PHR field has a length of 1 byte, where the Most Significant Bit (MSB) is reserved and the other 7 bits are used to specify the frame length information, enabling to have packets with a total length of 127 bytes;

- The PHY payload contains one field called PSDU that carries the data payload from the PPDU.

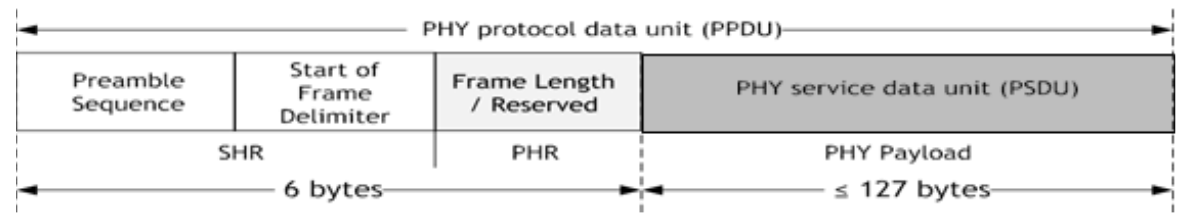

Figure 2. IEEE 802.15.4 PHY protocol data unit.

The IEEE 802.15.4 [4] PHY layer, (implemented in the OMNeT++ Network Simulation Framework [5]) operates in the $2.4 \mathrm{GHz}$ band and considers an O-QPSK modulation, offering extremely low BER performance, in the presence of a low Signal-to-Noise Ratio (SNR).

The BER is addressed by considering an interference signal similar to Additive White Gaussian Noise (AWGN). The radio transceivers operating in the $2.4 \mathrm{GHz}$ band employ a DSSS spread- ing technique. Therefore, the transmitter signal takes up more bandwidth than required to transmit the information signal being modulated. The name "spread spectrum" derives from the fact that the carrier signals occur over the full bandwidth (spectrum) of the sensor nodes transmitting frequency band. The modulation and spreading technique uses a 16-ary quasi-orthogonal modulation, where four information bits are used to select one of 16 nearly orthogonal Pseudo-Random Noise (PN) sequences to be transmitted. Therefore, a power-efficient modulation method that achieves low SNR and Signal-to-Interference Ratio (SIR) requirements is used.

The O-QPSK modulation uses a chip rate, $R_{c}$, of $2 \mathrm{Mchip} / \mathrm{s}$ and a bit rate, $R_{b}$, of $250 \mathrm{~kb} / \mathrm{s}$, by using a codebook of $M=16$ symbols. The conversion from SNR to the noise density, $\left(E_{b} / N_{0}\right)$ assumes a matched filtering and a half-sine pulse shaping, as shown in equation (1):

$$
\frac{E_{b}}{N_{0}}=\frac{0.625 \cdot R_{c}}{R_{b}}=\frac{0.625 \cdot 2000000}{250000} \cdot S N R=5 \cdot S N R
$$

Equation (2) presents the conversion of these ratios from bit noise density, $E_{b} / N_{0}$, to symbol noise density, $E_{s} / N_{0}$ :

$$
\frac{E_{s}}{N_{0}}=\log _{2}(M) \cdot \frac{E_{b}}{N_{0}}=4 \cdot \frac{E_{b}}{N_{0}}
$$

As shown in [6], the probability of Symbol Error Rate (SER), $P_{s}$, is computed by using the following equation:

$$
P_{S}=\frac{1}{M} \cdot \sum_{j=2}^{M}(-1)^{j}\left(\begin{array}{c}
M \\
j
\end{array}\right) \cdot e^{\left(\frac{E_{S}}{N_{0}}\left(\frac{1}{j}-1\right)\right)}
$$

Finally, for an $M$-ary orthogonal signal, the conversion from $P_{s}$ to the probability of bit error, $P_{b}$, is given by:

$$
P_{b}=P_{s}\left(\frac{M / 2}{M-1}\right)=P_{s}\left(\frac{8}{15}\right)
$$


By combining Equations (1) to (4), one obtains the BER function:

$$
B E R=\left(\frac{8}{15}\right) \frac{1}{M} \cdot \sum_{j=2}^{M}(-1)^{j}\left(\begin{array}{c}
M \\
j
\end{array}\right) \cdot e^{\left(\frac{E_{s}}{N_{0}}\left(\frac{1}{j}-1\right)\right)}
$$

Since the modulation and spreading technique consists of using a 16-ary quasi-orthogonal modulation technique, one obtains the following equation for $B E R$.

$$
B E R=\left(\frac{8}{15}\right)\left(\frac{1}{16}\right) \cdot \sum_{j=2}^{16}(-1)^{j}\left(\begin{array}{c}
16 \\
j
\end{array}\right) \cdot e^{\left(20 \cdot \operatorname{SNR} \cdot\left(\frac{1}{j}-1\right)\right)}
$$

Figure 3 presents the BER as a function of the SNR for both SBACK-MAC and IEEE 802.15.4 (by considering the 2.4 GHz band). The Signal-to-Interference-plus-Noise Ratio (SNIR) values correspond to the BER lower bound and are lower than $1 \times 10^{-8}$. In our simulator, this value is used as a parameter for the network.

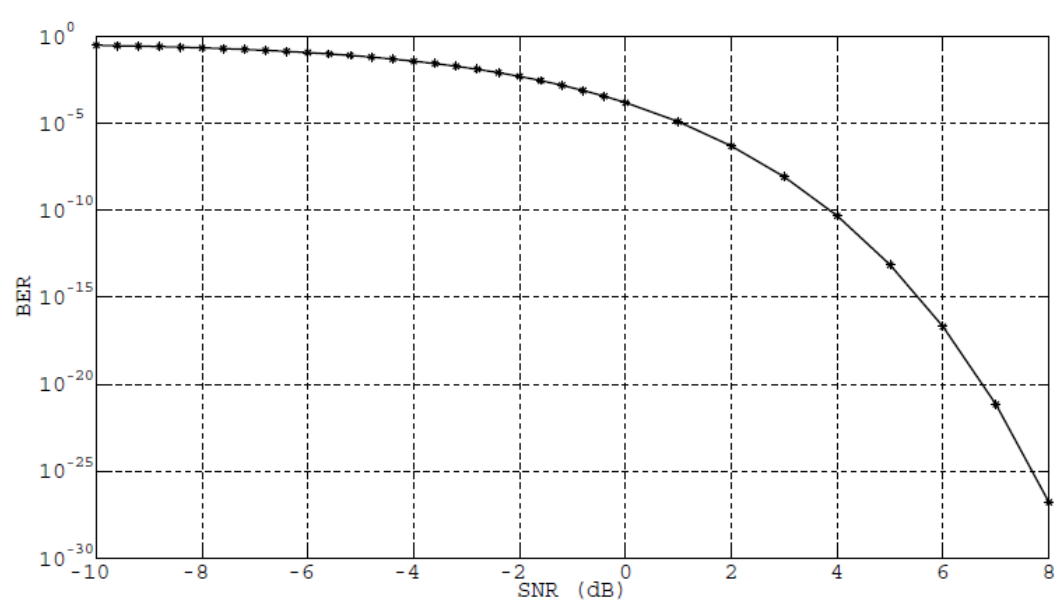

Figure 3. BER as a function of the SNR for IEEE 802.15.4 and RTS/CTS combined with packet concatenation.

The Packet Success Rate (PSR), is defined in [7] as the probability that the frame has no errors, and is given by:

$$
P S R=(1-B E R)^{(n B i t s-1)}
$$

where $n$ Bits is the length of the frame, in bits.

The Packet Error Rate (PER) for a given packet is given by:

$$
P E R=1-P S R
$$

Figure 4 shows the PERs for different SNRs by considering 2 different data packets lengths with data payload of 118 bytes and 18 bytes respectively, and an ACK control packet with length of 11 bytes. 


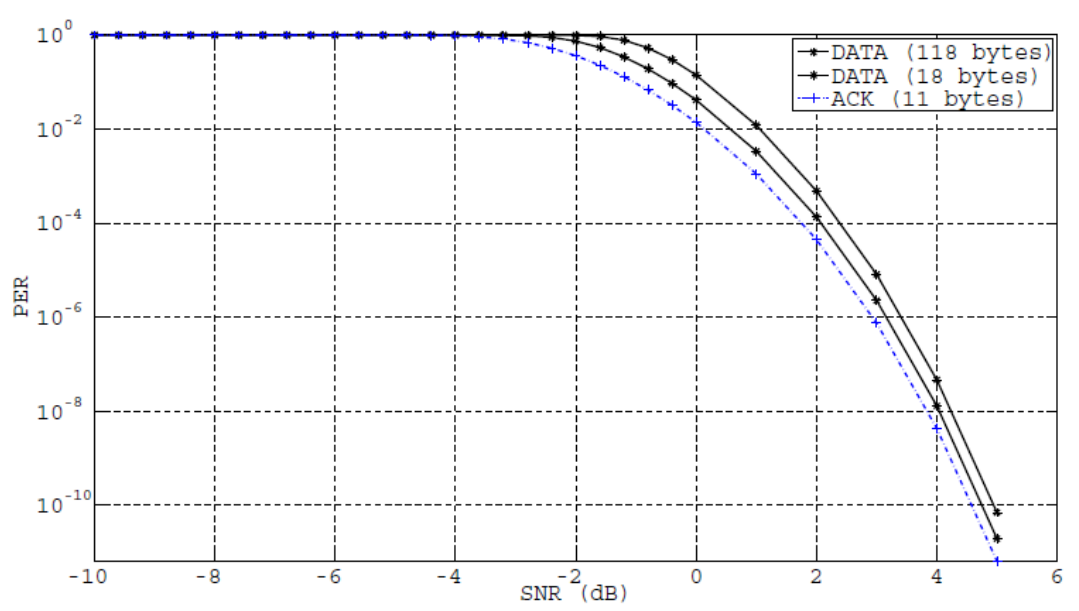

Figure 4. PER as a function of the SNR for IEEE 802.15.4 and RTS/CTS. and RTS/CTS combined with packet concatenation.

By analyzing Equation (8) and Figure 4, one concludes that, by increasing the SNR, the ratio between the received power and noise is increased, so that the PER decreases. Moreover, for longer packet lengths (i.e., for data packets) the PER is higher. In [8], authors have proposed a reliability based decider to improve the packet reception rate at the PHY layer which is characterized by metrics like the SNIR, BER, SER,PER, and outage probability, whose implementation in the MiXiM framework of the OMNeT++ simulator is considered here. With the appearance of more and more wireless devices operating in the $2.4 \mathrm{GHz}$ industrial, scientific and medical (ISM) band portion of the radio spectrum, there was an increase of the signals from other interfering sources. Moreover, in most of the cases, there is no collaboration between these independent wireless networks, and the operation of one network may adversely affect the other ones. Hence, there is a need for allowing a better coexistence between the different wireless devices operating in the same frequency band, whilst ensuring QoS. In order to cope with the design requirements and allow enhanced coexistence between the existing 2.4 GHz systems, like Cordless Phones, Bluetooth, Wi-Fi (i.e., IEEE 802.11b/g/n) and IEEE 802.15.4, MAC management efficient techniques must be addressed allowing for mitigating the interference from other sources under hostile conditions. Figure 5 presents the utilized channel bandwidth as well as the expected output power for each wireless system.

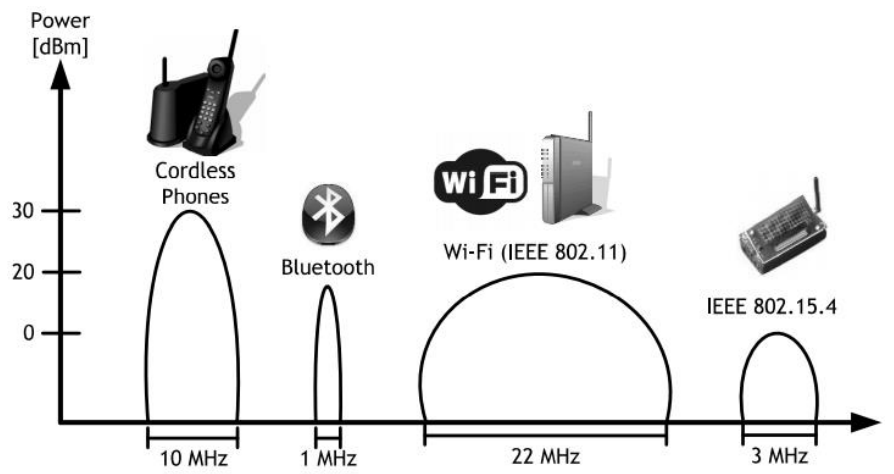


Figure 5. Wireless network technologies operating in the $2.4 \mathrm{GHz}$ ISM frequency band.

Figure 6 presents the IEEE 802.15.4 and IEEE $802.11 \mathrm{~b} / \mathrm{g} / \mathrm{n}$ channel overlapping in detail for the $2.4 \mathrm{GHz}$ ISM band.

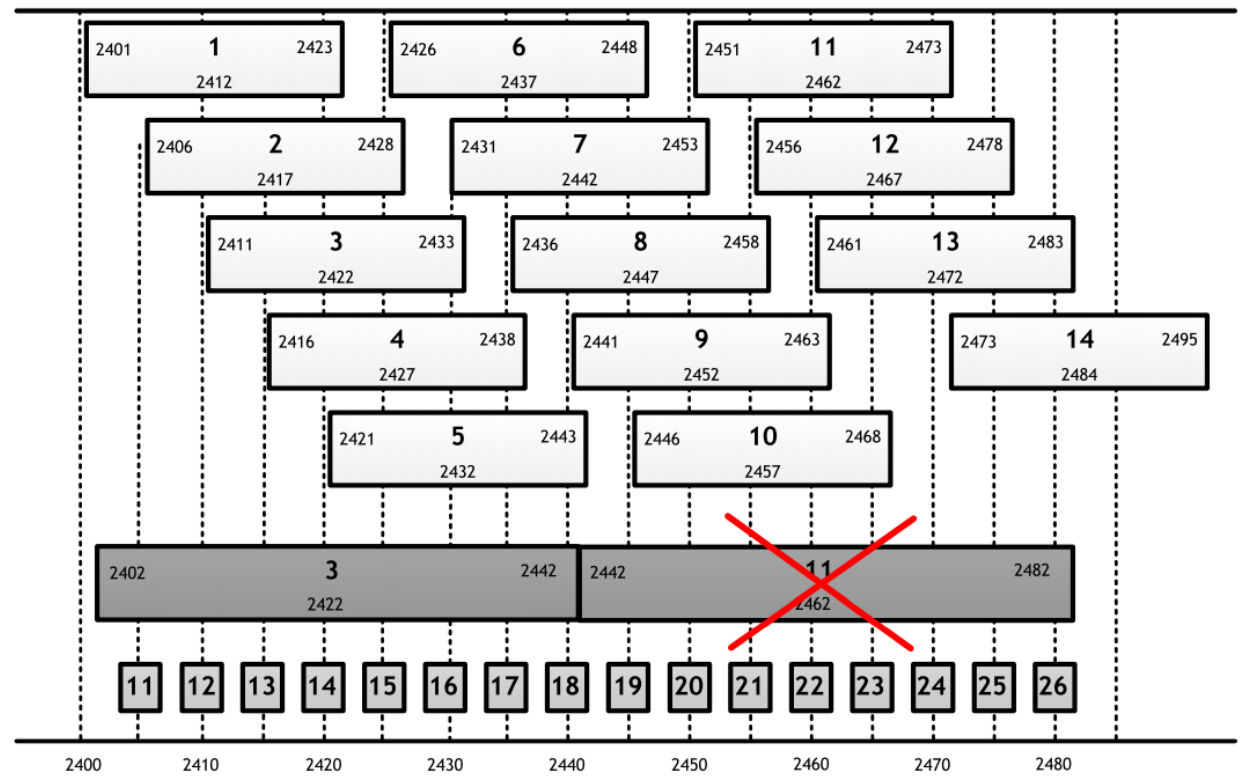

Figure 6. EEE 802.15.4 and IEEE $802.11 \mathrm{~b} / \mathrm{g} / \mathrm{n}$ channel overlapping in the $2.4 \mathrm{GHz}$ ISM band.

The IEEE 802.15.4 frequency channels 15, 20, 25, and 26 are the ones that will suffer the least interference from IEEE 802.11b/g in North America compared to other IEEE 802.15.4 frequency channels. In Europe the IEEE 802.15.4 channels that will suffer the least interference from IEEE $802.11 \mathrm{~b} / \mathrm{g}$ are the channels $15,16,21$ and 22 .

\section{MAC Sub-layer}

In the IEEE 802.15.4 [4] basic access mode, nodes use a nonbeacon-enabled CSMA-CA algorithm for accessing the channel and transmit their packets. The unslotted Carrier Sensing Multiple Access - Collision Avoidance (CSMA-CA) in the nonbeacon-enabled mode will facilitate a better flexibility for large-scale IEEE 802.15.4compliant peer-to-peer networks [9]. Before, each transmission the MAC sub-layer exchange messages with the PHY layer for packet transmission (TX)/reception (RX). Figure 7 presents the algorithms flowchart showing the interaction between the different packet types (e.g., DATA and ACK) and the control messages, being invoked when a packet is transmitted/received.

The PHY and MAC layers exchange control messages every time an event occurs as follows:

\section{PHY -> MAC}

- RX_START: Start of message indicator;

- RX_FAIL: Failed to receive message after RX_START. The message can fail because Cyclic Redundancy Check (CRC) or collision;

- $\quad$ TX_END: Message being transmitted has completed; 
- TX_FAIL: End of transmission (like TX_END) but the message transmission has failed. For most radio transceivers this should never happen (but there are valid cases for packet-based radios, e.g., CC2420).

After starting carrier sense, one of the following messages must be sent to the MAC layer as follows:

- CHANNEL_IDLE: If the specified "packet length" has been processed, and the carrier sense returns channel not "busy";

- CHANNEL_BUSY: If the carrier sense returns channel "busy".
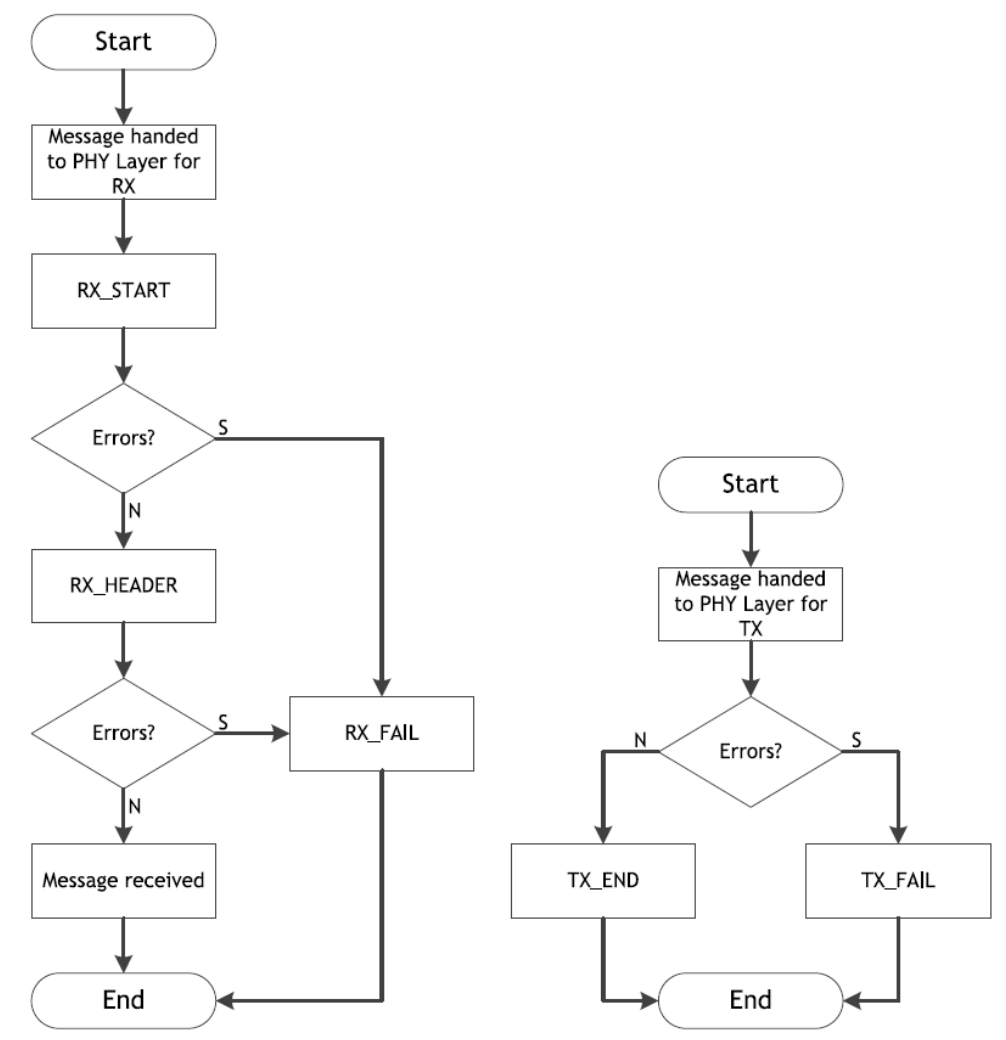

Figure 7. Control messages flowchart for the nonbeacon-enabled mode of IEEE 802.15.4.

\section{MAC -> PHY}

- $\quad$ SET_TRANSMIT: Switch the PHY layer to the transmit mode;

- $\quad$ SET_LISTEN: Switch the PHY layer to the listen mode;

- $\quad$ SET_SLEEP: Switch the PHY layer to the sleep mode;

- $\quad$ START_CARRIER_SENSE: Start carrier sense.

When using slotted CSMA-CA, each operation (channel access, backoff counter and CCA) can only occur at the boundary of a backoff period (BP). Additionally, the BP boundaries must be aligned with the slot boundaries of the superframe time [10]. In non-slotted CSMA-CA the backoff periods of one node are completely independent of the backoff periods of any other node in a PAN. 
The backoff phase (N.B., this time period is not generally called contention window in IEEE 802.15.4) algorithm is implemented by considering basic units of time called backoff periods. The backoff period duration is equal to $T_{B O}=20 \times T_{\text {symbol }}$ (i.e., $0.32 \mathrm{~ms}$ ), where $T_{\text {symbol }}=16 \mu \mathrm{s}$ is the symbol time [4]. Before performing CCA, a device shall wait for a random number of backoff periods, determ- ined by the backoff exponent (BE). Then, the transmitter randomly selects a backoff time period uniformly distributed in the range $\left[0,2^{B E}-1\right]$. Therefore, it is worthwhile to mention that even if there is only one transmitter and one receiver, the transmitter will always choose a random backoff time period within $\left[0,2^{B E}-1\right]$. Initially, each device sets the $B E$ equal to macMinBE, before starting a new transmission and increments it, after every failure to access the channel. In this work we assume that the $B E$ will not be incremented since we are assuming ideal conditions.

Table 2 from [11] summarizes the key parameters for both the IEEE 802.15.4 standard employing or not employing RTS/CTS with frame concatenation protocol for the $2.4 \mathrm{GHz}$ band, by considering the DSSS PHY layer with the O-QPSK modulation.

IEEE 802.15.4 [4] nodes support a maximum over-the-air data rate of $250 \mathrm{~kb} / \mathrm{s}$. However, in practice, the effective data rate is lower due to the protocol timing specifications, [4]. This can also be explained by the various mechanisms that are employed to ensure robust data transmission, including channel access algorithms, data verification and frame acknowledgement. In this work we address unicast data transmissions with ACKs, being the channel access time a dominant factor in the overall performance of the network. The regular procedure of the IEEE 802.15.4 nonbeacon-enabled mode is presented in Figure 8. When a device wishes to transfer data, it simply transmits its data frame, using unslotted CSMA-CA, to the coordinator. The coordinator acknowledges the successful reception of the data by transmitting an ACK control packet.

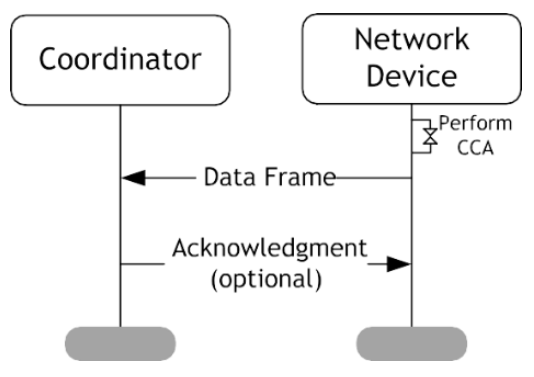

Figure 8. IEEE 802.15.4 - Communication to a coordinator in a nonbeacon-enabled PAN.

In this research work, we only consider the nonbeacon-enabled mode. The beacon-enabled mode is not considered because collisions can occur between beacons or between beacons and data or control frames, making a multi-hop beacon-based network difficult to be built and maintained [12]. Another important attribute is scalability, an intrinsic characteristic of multi-hop WSNs. Changes in terms of network size, node density and topology may occur. Nodes may die over time. Other nodes may be added later, and some may move to different locations. Therefore, for such kind of networks, the nonbeacon-enabled mode seems to be more 
adapted to the scalability requirement than the beacon-enabled mode. In the former case, all nodes are independent from the PAN coordinator and the communication is completely decentralized.

Moreover, for beacon-enabled networks [4], there is an additional timing requirement for sending two consecutive frames, so that the ACK frame transmission should be started between the TX/RX or RX/TX switching time, $T_{T A}$, and $T_{T A}+T_{B O}$ time periods (and there is time remaining in the Contention Access Period (CAP), for the message, appropriate interframe space, Interframe Space (IFS) and ACK). Figure 9 presents the timing requirements for transmitting a packet and receiving an ACK for the beacon and nonbeacon-enabled modes, respectively.

In IEEE 802.15.4 [4], [10], the CSMA-CA algorithm is significantly different from the one used in IEEE 802.11e [13]. The main differences are related to the backoff algorithm. In IEEE 802.11e [13], the value of the Contention Window $(\mathrm{CW})$ depends on the number of failed retransmissions for the packet, whereas, in the basic access mode for IEEE 802.15.4, this value (denoted as backoff phase) depends on the backoff exponent (BE), and number of backoffs (NB). Moreover, in IEEE 802.11e, the backoff time counter (BOc) is decreased as long as the channel is sensed idle and is frozen when a transmission occurs. In the IEEE 802.15.4 basic access mode, nodes do not continuously monitor the channel during the backoff phase and the sensing phase (i.e., CCA) only occurs at the end of the backoff phase.

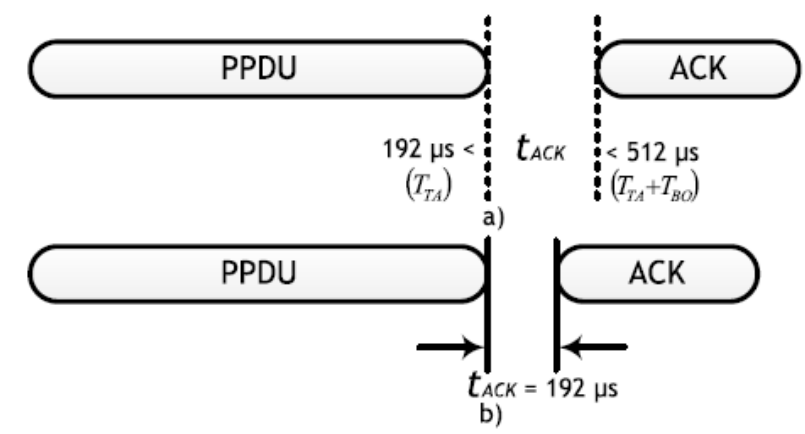

Figure 9. IEEE 802.15.4 acknowledgment frame timing: a) beacon and b) nonbeacon-enabled modes.

According to the IEEE 802.15.4 standard [10], a sensor node that sends a data or a MAC command frame with its ACK Request subfield set to one shall wait for at most an ACK wait duration period, TAW , for the corresponding ACK frame to be received. The $T_{A W}$ already includes the time for the ACK frame itself. The transmission of an ACK frame in a nonbeacon- enabled PAN or in the Contention Free Period (CFP) shall start aTurnaroundTime symbols (i.e., $192 \mu \mathrm{s}$ ) after the reception of the last symbol of the DATA or MAC command frame ([10], Section 7.5.6.4.2).

The ACK wait duration period, $T_{A W}$, is calculated as follows:

$$
T_{A W}=T_{\text {Symbol }}+T_{T A}+T_{S H R}+\left[6 \times T_{\text {Symbol }} \times \text { phySymbolsPerOctect }\right]
$$

By considering the DSSS PHY layer for the $2.4 \mathrm{GHz}$ band, the maximum ACK wait duration period, $T_{A W}$, is given by: 


$$
T_{A W}=16 \mu s+192 \mu s+160 \mu s+192 \mu s=560 \mu s
$$

Figure 10 presents the ACK timing required for the IEEE 802.15.4 standard, by considering the DSSS PHY layer for the $2.4 \mathrm{GHz}$ band at $250 \mathrm{~kb} / \mathrm{s}$. The receivers start transmitting the ACK, $192 \mu$ s (i.e., TTA) after the reception of the DATA frame. By assuming a DATA and an ACK frame with 18 and 11 bytes, respectively (including the PHY and MAC overhead), the transmission time is $576 \mu$ s and $352 \mu$ s, respectively. As reference, Figure 7 also includes the ACK wait duration period, $T_{A W}$.

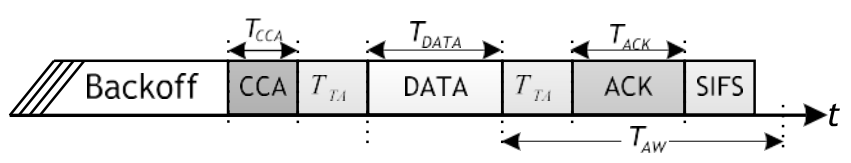

Figure 10. Acknowledgement process timing.

For every DATA packet transmitted, there is a random deferral time period, $D_{T}$, before transmitting, which is given by:

$$
D_{T}=\text { InitialbackoffPeriod }+ \text { ccaTime }+T_{T A}
$$

The initial backoff period, Initialbackoff Period, is given as follows:

$$
\text { InitialbackoffPeriod }=C W_{N B}=\left(2^{B E}-1\right) \times T_{B O}
$$

The time delay, due to CCA, is given by:

$$
\text { ccaTime }=\text { rxSetupTime }+T_{C C A}
$$

The rxSetupTime is the time to setup the radio from a previous state to the transmission or reception states and it mainly depends on the radio transceiver used. During the $T_{C C A}$, the radio transceiver must determine the channel state within 8 symbol duration (i.e., $128 \mu$ s, which corresponds to one symbol duration of $16 \mu \mathrm{s}$ ). In a normal transmission, for every DATA packet sent an ACK must be received, as shown in Figure 11.

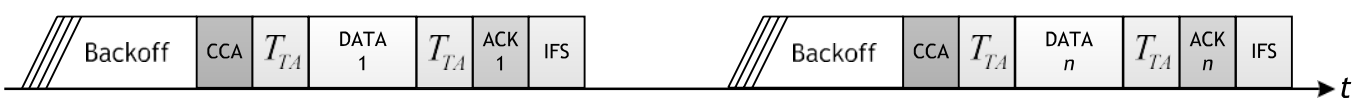

Figure 11. Acknowledgement process timing within the IEEE 802.15.4 basic access mode.

Details on the analytical model for the maximum throughput and minimum delay are given in [11].

\section{Brief overview of the MAC sub-layer system model}

The main reasons why IEEE 802.15.4 basic access mode does not consider the adoption of the RTS/CTS handshake mechanism are the following ones. (i) The introduction of RTS/CTS packets adds additional protocol overhead and, in a situation with low traffic load, short packet sizes could have the same order of magnitude of a RTS/CTS packet, (ii) The absence of a RTS/CTS handshake mechanism allows to reduce the system complexity. Although these assumptions are true for some particular cases, we argue that in the presence of link 
layer errors the additional protocol overhead due to the use of RTS/CTS packets is mitigated by our proposed concatenation mechanism.

In our proposal, we assume that both the RTS and CTS packets have the structure of an ACK packet, which is assumed to have a limited size of 11 bytes, as shown in Table 2 from [9]. The maximum data payload for IEEE 802.15.4 depends on the application (maximum payload could range between 102 and 118 bytes). Therefore, the length of the data packets could be approximately ten times larger than the control packets length. In reality, IEEE 802.15.4 employing RTS/CTS with packet concatenation is composed by the following time periods: backoff phase, CCA mechanism, time needed for switching from receiving to transmitting, RTS transmission time, time needed for switching from transmitting to receiving and CTS reception time.

Both the IEEE 802.15.4 basic access and the proposed RTS/CTS schemes consider acknowledgment (ACK) frames to confirm successful frame reception. Aiming at overhead reduction, the use of RTS/CTS frames enables channel reservation and avoids the replication of the backoff phase for every consecutive transmitted frame and implies zero backoff exponential. Moreover, by considering RTS/CTS, nodes avoid frame collisions, which often take place due to the hidden terminal problem. Hence, 802.15.4 performance is considerably enhanced, since the number of retransmitted frames is significantly decreased.

In reality, authors from [14] have demonstrated that one fundamental reason for IEEE 802.15.4 MAC inefficiency is overhead, originated, e.g., by inter-frame spaces from the protocol, backoff period, transmission of PHY/MAC headers and ACKs, interference and retransmissions (due to unsuccessful reception of data frames). The un-slotted Carrier Sense Multiple Access with Collision Avoidance (CSMA/CA) algorithm and the backoff phase are characterized by using the formulation presented in [15]. N.B.: we designate the backoff phase by Contention Window $(\mathrm{CW})$.

To determine the maximum average throughput for the basic access mode, $S_{\max }$, the minimum average delay, $D_{\min }$ is first derived. Figure 12 presents the frame structure for the IEEE 802.15.4 basic access mode in the absence of RTS/CTS while considering retransmissions [14]. As mentioned above, under IEEE 802.15.4 employing RTS/CTS and frame concatenation, nodes use the same backoff procedure as in 802.15.4 but only for each RTS/CTS set. Therefore, the channel utilization is enhanced by decreasing the deferral time before transmitting each data frame, as shown in Figure 13.

The minimum delay due to Clear Channel Assessment (CCA), $D_{\text {min_CCA_RTS, }}$ (enabled to estimate if the channel state is busy or idle after the backoff phase) and prior to each RTS/CTS set is given by:

$$
D_{\text {min_CCA_RTS }}=\sum_{i=1}^{n / N_{\text {agg }}} \sum_{k=0}^{k \leq N B}\left(\overline{C W_{k}}+\text { ccaTime }\right),
$$

As in [15], the number of backoff periods is given by $N B \in\left[0, N B_{\max }\right]$. 


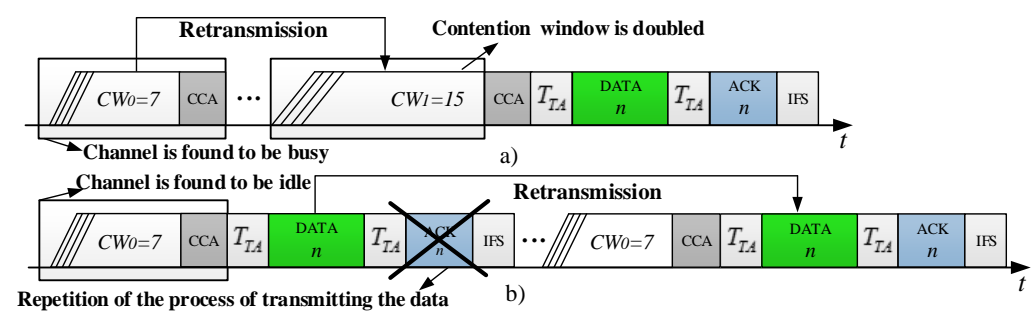

Figure 12. IEEE 802.15.4 basic access mode with retransmissions when channel is assessed as a) busy and b) idle.

The time delay due to CCA is given by ccaTime $=$ rxSetupTime $++T_{C C A}$, where rxSetupTime is the time to switch the radio between different states. Equation (14) considers that nodes only determine the channel state once per RTS/CTS exchange. So, if the total number of transmitted frames in a node is $n=100$ and the number of aggregated frames is $N_{a g g}=10$, it only determines the channel state $n / N_{a g g}=10$ times, i.e., once per exchange of RTS/CTS, plus the time needed for transmitting the frames (until the maximum limit for the number of retries, $N B_{\max }=4$, is reached).

If the estimation of the channel is idle during CCA and, if after sending a data frame an ACK is not received within a duration equal to $T_{A W}$, the retransmission process does not consider a backoff phase between two consecutive data frames. This simplification decreases the total overhead, as shown in Figure 13. Since any other station will receive all RTS/CTS/DATA/ACK frames, in the first transmission attempt, it will set the Network Allocation Vector (NAV). The NAV is responsible for defining the time duration for channel access deferring in order to avoid collisions.

With erroneous channels under IEEE 802.15.4 employing RTS/CTS, if the channel estimation is idle during CCA, there is data transmission and an ACK is not received within a duration of $T_{A W}$, the delay due to frame retransmissions RTXs), is given by:

$$
D_{\text {minDataRetRTS }}=\left\{\begin{array}{lc}
H_{1}, & \text { for } j=0 \\
H_{2}, & \text { for } j \in[1, \text { MaxRet }]
\end{array},\right.
$$

where $j$ is the number of RTXs, varying between 1 and MaxRet [1].

From the analysis of equation (1) one can conclude the following:

(a) after CCA, if a node determines that the channel is found to be idle and an ACK is correctly received for each sent frame, the minimum delay, $D_{\text {minDataRetRTS }}$, is determined by:

$$
\begin{aligned}
H_{1}=T_{T A}+ & T_{R T S}+T_{T A}+T_{C T S}+\ldots+ \\
& +\sum_{i=1}^{N_{\text {agg }}}\left(\text { ccaTime }+T_{T A}+T_{D A T A}+T_{T A}+T_{A C K}+T_{I F S}\right),
\end{aligned}
$$

where $T_{T A}$ is the TX/RX or RX/TX switching time. $T_{D A T A}, T_{A C K}$, and $T_{I F S}$ are the durations of the data frame, ACK frame and inter-frame spacing (IFS), respectively. Since transmission errors do not exist, the number of retransmissions is $j=0$. As such, in equation (16), there is no need to consider the ACK wait duration period, $T_{A W}$, which represents the longest time needed to receive an ACK control frame. 
(b) After CCA, if a node estimates that channel is idle and an ACK has not been received within the duration $T_{A W}$, for one or more transmitted frames (since we consider frame concatenation), the minimum delay due to

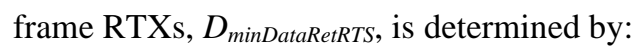

$$
\begin{aligned}
& H_{2}=T_{T A}+T_{R T S}+T_{T A}+T_{C T S}+\ldots+\sum_{i=1}^{N_{a g g}-m}\left(\text { ccaTime }+T_{T A}+T_{D A T A}+\right. \\
& \left.+T_{T A}+T_{A C K}+T_{I F S}\right)+\sum_{i=1}^{m}\left(j_{i} \cdot\left(\text { ccaTime }+T_{T A}+T_{D A T A}+T_{A W}\right)\right)+ \\
& +\sum_{i=1}^{m}\left(\text { ccaTime }+T_{T A}+T_{D A T A}+T_{T A}+T_{A C K}+T_{I F S}\right)
\end{aligned}
$$

The term $\sum_{i=1}^{N_{a g g}-m}\left(\right.$ ccaTime $\left.+T_{T A}+T_{D A T A}+T_{T A}+T_{A C K}+T_{I F S}\right)$ represents the duration of the $N_{a g g}-m$ transmitted frames whose ACK response was successful, where $m$ denotes the number of transmitted (TX) frames that need retransmission. Due to lack of ACK frame reception, each individual frame can be retransmitted more than once.

The term $j_{i}$ represents the number of RTXs until MaxRet has been reached. The last term corresponds to successful reception of the ACK.

The minimum average delay, $D_{\text {min_RTS }}$, accounting for channel state and frame RTXs is obtained by combining equations (14)-(17):

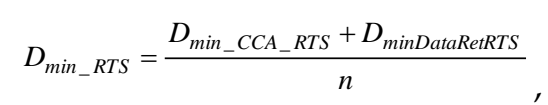

The maximum average throughput, by considering frame RTXs, $S_{\max \_ \text {RTS }}$, in bits per second, is then given by:

$$
S_{\text {max_RTS }}=8 \cdot L_{D A T A} / D_{\text {min_}_{-} R T S} \text {, }
$$

\section{Analytical and Simulation Results}

We have compared IEEE 802.15.4 employing and not employing RTS/CTS by using the MiXiM framework of the OMNeT++ simulator [16]. A two-hop network, with two sources nodes, one relay and two sink nodes has been considered. Two interferers are responsible for sending broadcast frames that collide with the frames sent by the sources and central node. The DSSS and CSS PHY layers performance analysis considers several runs with five different random seeds and a $95 \%$ confidence interval. A perfect match between analytical and simulation results was obtained.

Table 1 compares channel access times and overhead for the DSSS and CSS PHY. Respective maximum data rates are $250 \mathrm{~kb} / \mathrm{s}$ and $1 \mathrm{Mb} / \mathrm{s}$ [1]. Likewise, in [14], we analyze both $S_{\max }$ and $D_{\min }$.

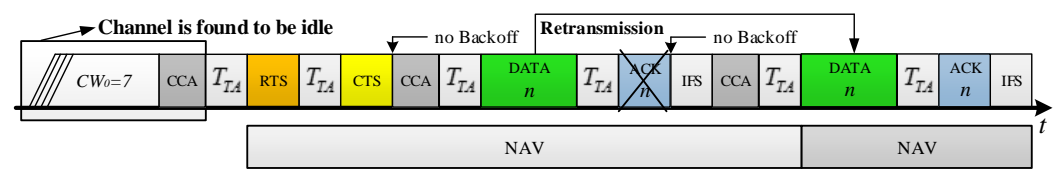

Figure 13. IEEE 802.15.4 with RTS/CTS and retransmissions. 
Table 1. Comparison of the values and time parameters between the DSSS and CSS PHY layers for IEEE 802.15.4 (2.4 GHz Band).

\begin{tabular}{ccc}
\hline Symbol & DSSS PHY & CSS PHY \\
\hline$L_{H_{-} P H Y} / L_{H_{-} M A C}$ & 6 bytes $/ 9$ bytes & 7 bytes $/ 9$ bytes \\
$T_{T A} / T_{B O}$ & $192 \mu \mathrm{s} / 320 \mu \mathrm{s}$ & $72 \mu \mathrm{s} / 120 \mu \mathrm{s}$ \\
$T_{\text {SIFS }} / T_{\text {LIFS }}$ & $192 \mu \mathrm{s} / 640 \mu \mathrm{s}$ & $72 \mu \mathrm{s} / 240 \mu \mathrm{s}$ \\
$R$ & $250 \mathrm{~kb} / \mathrm{s}$ & $1 \mathrm{Mb} / \mathrm{s}$ \\
\hline
\end{tabular}

Although we consider the $2.4 \mathrm{GHz}$ band, the proposed formulation can be also applied to other frequency bands. A fixed payload size $L_{D A T A}=3$ bytes is considered. In [17], authors proved that, for short frame sizes, IEEE 802.15.4 achieves poor performance. Here, it is shown that the proposed mechanism can significantly improve channel efficiency even with retransmissions (RTX of $10 \%$ of packets is assumed).

Figures 14,15 and 16 present $D_{\min }, S_{\max }$ and the bandwidth efficiency, $\eta$, as a function of the number TX frames [17] for the DSSS/CSS PHY:

$$
\eta=S_{\max } / R
$$

where $R$ represents the maximum data rate.

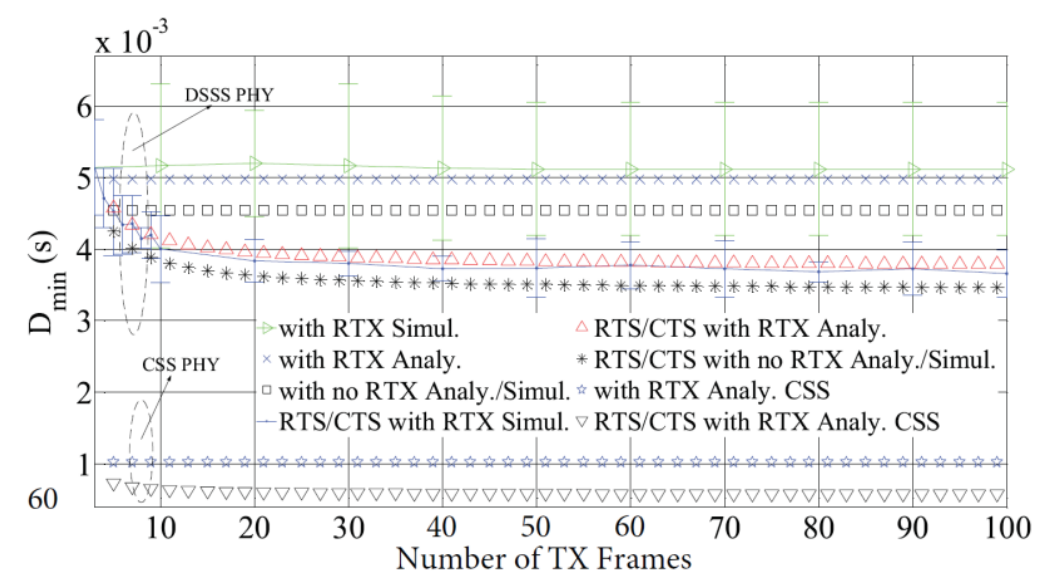

Figure 14 Minimum average delay versus the number of TX frames for the basic access and RTS/CTS modes.

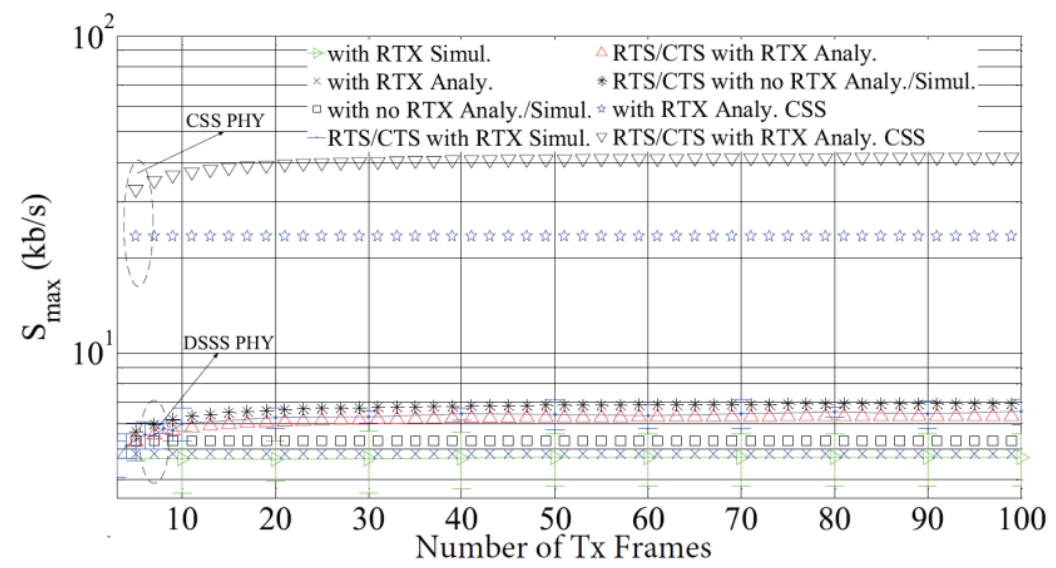

Figure 15. Maximum average throughput versus the number of TX frames for basic access and RTS/CTS modes. 


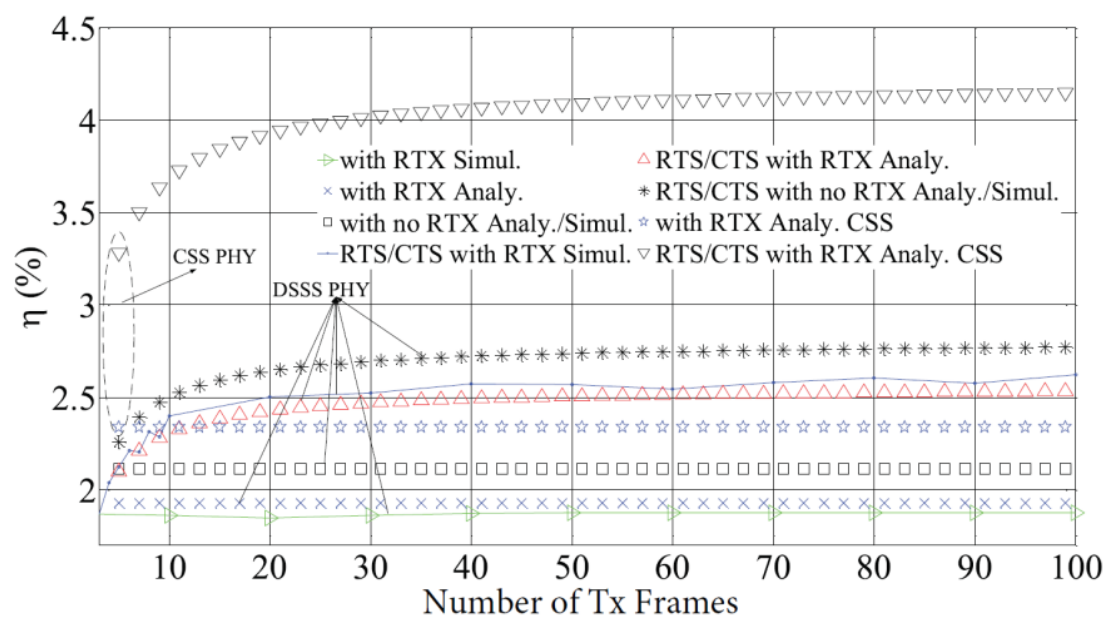

Figure 16. Bandwidth efficiency versus the number of TX frames for IEEE 802.15.4 basic access and RTS/CTS modes.

Results show the global underperformance of the basic access mode of 802.15.4 without employing RTS/CTS compared to the use of RTS/CTS and frame concatenation, regardless the use of frame RTXs, in terms of $D_{\text {min }}$, $S_{\max }$ and $\eta$ for both PHY layers. The number of TX frames varies between 1 and 100. The performance of CSS is clearly better than the one of the DSSS PHY layer. Moreover, performance results for $D_{\min }$ as a function of the number of TX frames show that, for the DSSS PHY layer, by considering RTS/CTS with frame concatenation, for 5 and 10 aggregated frames, $D_{\min }$ decreases $\left(S_{\max }\right.$ increases) $8 \%$ and $18 \%$, respectively. For more than 28 aggregated frames, $D_{\min }$ decreases $\left(S_{\max }\right.$ increases) $\sim 30 \%$. For the CSS PHY layer, by using RTS/CTS with frame concatenation, for 5 and 10 aggregated frames, $D_{\min }$ decreases ( $S_{\max }$ increases) $33 \%$ and $59 \%$, respectively. For more than 28 aggregated frames, $D_{\min }$ decreases $\left(S_{\max }\right.$ increases $) \sim 71 \%$.

\section{Conclusions}

This paper proposes a retransmission model for the non-beacon-enabled mode of IEEE 802.15.4 that employs RTS/CTS and frame concatenation, where the backoff procedure is not repeated for each data frame sent, but only once for each RTS/CTS set. Performance results clearly show the substantial benefits of using RTS/CTS, in terms of maximum throughput, minimum delay and bandwidth efficiency, in particular for the CSS PHY layer, as the proposed MAC sub-layer protocol shows a clear reduction in delay, enhancement in maximum average throughput, and improved bandwidth efficiency, from $\sim 2.5 \%$ to $\sim 4.2 \%$ when the CSS PHY is considered. The study of the energy efficiency of the proposed MAC sub-layer enhancement is left for further study.

Author Contributions: Conceptualization, Norberto Barroca, Fernando J. Velez, and Periklis Chatzimisios; methodology, Norberto Barroca, Fernando J. Velez, Luís M. Borges and Periklis Chatzimisios; software, Norberto Barroca, Fernando J. Velez and, Luís M. Borges; validation, Norberto Barroca, Fernando J. Velez.; writing - original draft preparation, Norberto Barroca, Fernando J. Velez, and Periklis Chatzimisios; writing review and editing, Norberto Barroca, Fernando J. Velez, Luís M. Borges and Periklis Chatzimisios. All authors have read and agreed to the published version of the manuscript. 
Funding: This work has been partially supported and funded by the Ph.D. FCT grant FRH/BD/66803/2009, by CREaTION, ECOOP, UIDB/EEA/50008/2020, COST IC0905 TERRA, COST CA 15104, SFRH/BSAB/113798/2015, CONQUEST, TeamUp5G and ORCIP.

\section{References}

1. Wireless Medium Access Control and Physical Layer Specific. for Low-Rate Wireless Personal Area Networks, IEEE Std. 802.15.4, 2011.

2. 'IEEE 802.15.4 Working Group for WPAN', http://www.ieee802.org/15, accessed June 2016

3. Chatzimisios, P., Boucouvalas, A.C., Vitsas, V.: 'Effectiveness of RTS/CTS handshake in IEEE 802.11a Wireless LANs', IEE Electronics Letters, 2004, 40, (14), pp. 915-916, doi: 10.1049/el:20040510

4. Part 15.4: Low-Rate Wireless Personal Area Networks - Revision of IEEE Std 802.15.4-2006, IEEE Std. 802.15.4TM, Sep. 2011.

5. OMNeT++ Network Simulation Framework, Sep. 2013. [Online]. Available: http://www.omnetpp.org/.

6. Sklar, B.: Digital Communications: Fundamentals and Applications, Prentice Hall, New Jersey, 1988.

7. Karl, H., and Willig, A.: Protocols and Architectures for Wireless Sensor Networks, Wiley, Chichester, UK, 2005.

8. Borges, L.M., Velez, F.J., Barroca, N., and Lebres, A.S.: "Frame Capture and Reliability Based Decider Implementation in the MiXiM IEEE 802.15.4 Framework," in Proc. of International Conference on Simulation Tools and Techniques 2014, Lisbon, Portugal, Mar. 2014.

9. N. P. Mahalik: Sensor Networks and Configuration: Fundamentals, Standards, Platforms, and Applications, Springer, New York, USA, 2007.

10. Part 15.4: Wireless Medium Access Control and Physical Layer Specifications for Low-Rate Wireless Personal Area Networks, IEEE Std. 802.15.4TM, Sep. 2006.

11. Barroca, N., Borges, L.M., Velez, F.J., and Chatzimisios, P.: "Performance Enhancement of IEEE 802.15.4 by employing RTS/CTS with Packet Concatenation," IET Wireless Sensor Systems, vol. 10, no. 6, Dec. 2020, DOI: 10.1049/iet-wss.2019.0003, Print ISSN 2043-6386, Online ISSN 2043-6394.

12. Sun, M., Sun, K., and Zou, Y.: "Analysis and improvement for 802.15.4 multi-hop network," in Proc. of International Conference on Communications and Mobile Computing, Kunming, Yunnan, China, Nov. 2009, pp. 52-56.

13. Part 11: Wireless LAN Medium Access Control (MAC) and Physical Layer (PHY) specifications, IEEE Std. 802.11eTM, Nov. 2005.

14. Barroca, N., Borges L.M., Velez, F.J, Chatzimisios, C.: 'IEEE 802.15.4 MAC layer performance enhancement by employing RTS/CTS combined with packet concatenation', IEEE International Conference on Communications, Sydney, Australia, June 2014, pp. 466-471, doi: 10.1109/ICC.2014.6883362

15. Buratti, C., Verdone, R.: 'Performance Analysis of IEEE 802.15.4 Non Beacon-Enabled Mode', IEEE Trans. Vehicular Technology, 2009, 58, (7), pp. 3480-3493, doi: 10.1109/TVT.2009.2014956

16. 'MiXiM framework', http://sourceforge.net/apps/trac/mixim, accessed June 2016

17. Chowdhury, M.S., Ullah, N., Kabir H., Khan, P., Kwak, K.S.: 'Throughput, delay and bandwidth efficiency of IEEE 802.15.4a using CSS PHY', Int. Conf. on Information and Commun. Technology Convergence, 2010, pp. 158-163, doi: 10.1109/ICTC.2010.5674258 